\title{
Fluoreszenz und Phosphoreszenz im Lichte der neueren Atom-
} theorie. Von Peter Pringsheim. 2. Aufl. Verl. Jul. Springer, Berlin 1923. Preis 76.500 K.

Das Buch behandelt die seit dem Erscheinen des Kayserschen Handbuches der Spektroskopie Bd. IV (1908) veröffentlichten Arbeiten aus dem Gebiete der Photolumineszenz. Vier Kapiteln sind der Lumineszenz der Gase gewidmet und dabei auch. quantentheoretische Überlegungen zur Erklärung herangezogen. Die Resonanzstrahlung. (Linienfluoreszenz unter Reemission der erregenden Linie) beim $\mathrm{Hg}$ und $\mathrm{Na}$, ihr Übergang von der Volumresonanz zur Oberflächenresonanz und schließlich zur metallischen Reflexion wird besprochen, die spektrale Verteilung der Emission bei $\mathrm{J}$ und $\mathrm{Hg}$ sowie der Einfluß von Beimengungen. Von der Resonanzstrạhlung scharf getrennt ist die Bandenfluoreszenz der Gase, die einem veränderten (erregten) Z Z1stand der Molekiil zugeschrieben wird. $\mathrm{Hg}$ zeigt sowohl Resonanzstrahlung als Bandenfluoreszenz. Die Abklingungszeit der Gasfluoreszenz dürfte kleiner als $10^{-6}$ sein, wenn von dem Nachleuchten elektrisch erregter Fluoreszenz, das wohl auf andere Umstände zurückzuführen ist, abgesehen wird. Endlich werden die Polarisationsverhältnisse und der Einfluß von Magnetfeldern (Zeemanneffekt und Intensitätsverringerung) besprochen.

In den Kapitel über Lumineszenz der festen und flïssigen Lösungen wird dèr von Lenard geprägte Begriff der Lichtsumme eingeführt, als das Integral der von einem bestimmten Moment an bis zum völligen Ausleuchten ausgestrahlten Intensität, Diese Lichtsumme hat ein bei wachsender Erregung schließlich von Intensität und Dauer der Erregung unabhängiges Maximum: Die Frage nach dem Abklingungsgesetz der Phosphoreszenz, die spektrale Verteilung der erregten sowie des erregenden Lichtes, das Austreiben der Phosphoreszenz durch langwelliges Licht mit oder ohne Herabsetzung der Lichtsumme (Tilgung), der Zusammenhang der spektralen Lage der Phosphoreszenzbanden mit den Dielektrizitätskơnstanten, die Abhängigkeit der Absorption des erregenden Lichtes von Erregungszustand des Phosphors werden angeführt. Die letzten beiden Kapiteln behandeln die lichtstarken und in ihrer spektralen Verteilung durch ihre Gesetzmäßigkeiten so auffallenden Kristallfuoreszenzen besonders der Uranylsalze sowie die Lumineszenz der organischen Verbindungen. Hier zeigen sich, ähnlich wie bei den Erdalkaliphosphoren, neben rasch abklingenden Banden auch langsam abklingende (momentane und progressive). Das Literaturverzeichnis ist gegenuiber der ersten Auflage wesentlich ergänzt, doch fehlen z. B. die Arbeiten des Referenten.

F. Hotser.

\section{Atomabbau und Atombau. Die physikalische Analyse des} Atoms. Von Walter Gerlach, Jena, Gustav Fischer 1923. Preis $2 \mathrm{M}$. G. Z.

Die 50 Seiten starke Schrift, die Schönflies gewidmet ist, bildet eine Erweiterung einer Abhandlung, welche der Verfasser im Jahre 1922 in den Fortschritten der Mineralogie, Kristallographie und Petrographie erscheinen ließ. Über das Thema gibt es schon zahIreiche einführende und elementare gute Darstellungen. Trotz diesen ist die vorliegende Broschüre recht lesenswert; speziell die letzten Seiten enthalten verschiedenes, was man in andern Monographien nicht findet. $J$. Lewandowski.

Raum. Zeit. Materie. Vorlesungen über allgemeine Relativitätstheorie von H. Weyl. 5. Aufl. Mit 23 Textfiguren. VIII+ 338 Seiten. Berlin, Springer, 1923. Preis $10 \mathrm{M}$. (Grundzahl).

Der Verfasser hat eine neue Theorie der Materie aufgestellt, nach der die Materie keine Ausgeburt des.Feldes ist, sondern umgekehrt das Feld eindeutig bestimmt. Sie ist uiberhaupt nichts Räumliches.(Extensives), sondern steckt nur in einer bestimmten räumlichen Umgebung darin, als eine jenseits des Feldes liegende Singularität. Die Feldgesetze regeln die Wirkungsübertragung von Körper zu Körper. Dazu treten die tieferliegenden physikalischen Gesetze, nach denen die Materie die Feldzustände verursacht. Auf diesen Standpunkt stellt sich der Verfasser in der 